\title{
Performance Enhancement of a Dynamic Optical Code Division Multiple Access Communication System by Signal Clipping
}

\author{
Mohammad M. N. Hamarsheh, Mohamad K. Abdullah, Member, IEEE, M. A. Mahdi, S. Khatun, and \\ Hossam. M. H. Shalaby, Senior Member, IEEE
}

\begin{abstract}
An enhancement of a dynamic optical code division multiple access (DOCDMA) communication system is proposed by clipping the signal at the output of the decoder of each receiver. In a DOCDMA System, the central wavelength of a tunable optical filter (TOF) is dynamically varied according to a functional code at the transmitter during the bit period before the transmission of the data. The system is modeled and analyzed taking into account the multiple access interference, thermal noise, and phase induced intensity noise. The performance of the system with signal clipping is compared with that of no clipping and to a spectral amplitude coding system that uses either a Hadamard code or a modified quadratic congruence code. The results show that the proposed signal clipping method improves the bit error rate performance.
\end{abstract}

Index Terms-Multiple access interference, optical code division multiple access (CDMA), optical fiber communication.

\section{INTRODUCTION}

E arly optical CDMA systems coded the incoherent pulses in time domain and recovered the data at the receiver using taped delay lines. The performance of these direct sequence (DS) systems is poor because of the correlation properties of the special unipolar codes used and the summation required [1]. A more recent technique for optical CDMA systems uses spectral amplitude coding (SAC) [2]. In these systems, the spectrum of a broadband source is encoded. Further, the multiple access interference (MAI) can be canceled in these systems by using code sequences with fixed in-phase cross correlation. However, the phase induced intensity noise (PIIN) is the main parameter that limits the performance of this system [3]. The encoder for DS-CDMA systems is not reconfigurable and SAC needs special sophisticated reconfigurable encoder [4].

In this paper we propose an easily reconfigurable dynamic optical CDMA (DOCDMA) system with signal clipping. The encoder varies the central frequency of the

Manuscript received June 25,2005. This work was supported in part by the Malaysian Ministry of Science, Technology and Innovation, under IRPA Grant 02-02-04-T001.

Mohammad M. N. Hamarsheh, Mohamad K. Abdullah, M. A. Mahdi, and $\mathrm{S}$. Khatun are with the Department of Computer and Communication Systems Engineering, University Putra Malaysia, 43400, Serdang, Selangor, Malaysia. (e-mail: hamarsheh1@hotmail.com khazani@eng.upm.edu.my )

Hossam M. H. Shalaby is with the Department of Electrical Engineering, University of Alexandria, 21544 Alexandria, Egypt. (e-mail: shalaby@ieee.org) pulse optical signal according to a functional code. The synchronized system can recover the encoded data by a matched tunable optical filter at the receiver. DOCDMA signals interfere only during the time of intersection between the functional codes driving the TOF's. The system performance is better compared with the SAC systems recently proposed [2], [3]. Clipping the signal at the output of the detector reduces the effect of the MAI on the system and improves the performance. The encoder and decoder can be easily reconfigured to any of the functional codes without the need for any hardware modification.

\section{SYSTEM CONFIGURATION AND DESCRIPTION}

The block diagram in Fig. 1(a) shows the DOCDMA configuration. The broadband signal from the light source is OOK modulated with the binary data. For each data bit of "1", encoder $j, j \in\{1,2, \ldots K\}$, where $K$ is the number of simultaneous users, will filter the spectrum of the pulse at a central wavelength which varies according to a functional code $F^{j}(t)$. The encoder is simply a tunable optical filter controlled with the functional code. Signals transmitted from all synchronized users will be mixed up in the network before received by all users. At the receiver, the composite signal is decoded by matched tunable optical filter. Then, the signal passes through a photodetector, a clipper, an integrator, and a threshold decision to recover the data transmitted.

The source spectra are assumed to be flat over the bandwidth of $v_{0} \pm \Delta v / 2$, with magnitude $P_{r} / \Delta v$, where $v_{0}$ is the central optical frequency, $\Delta v$ is the system bandwidth, and $P_{r}$ is the received power from a single user. Ideal masking at the tunable optical filter is also assumed, and each user is considered to have the same effective average power at each receiver. The transmitter sends a pulse with spectral distribution varying with time if the data bit value is "1"; otherwise no power is transmitted. Fig. 1(b) shows the spectrum of $j^{\text {th }}$ user's transmitted signal when the data bit is "1". The spectrum is similar to that of an ideal filter with central frequency varying with time according to a functional code. The proposed functional codes family $F(t)$ is sine functions family with the same frequency and different phase shifts. Fig. 1(c) shows an example of the spectrum for two users at the input of the decoder during one bit period when both users are sending a bit of "1". The 


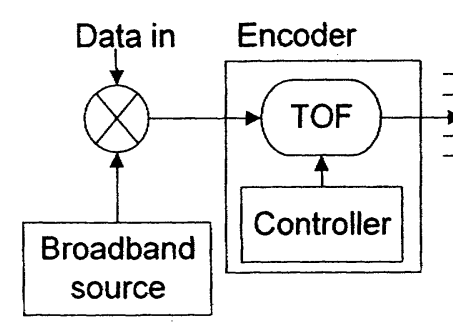

(a)
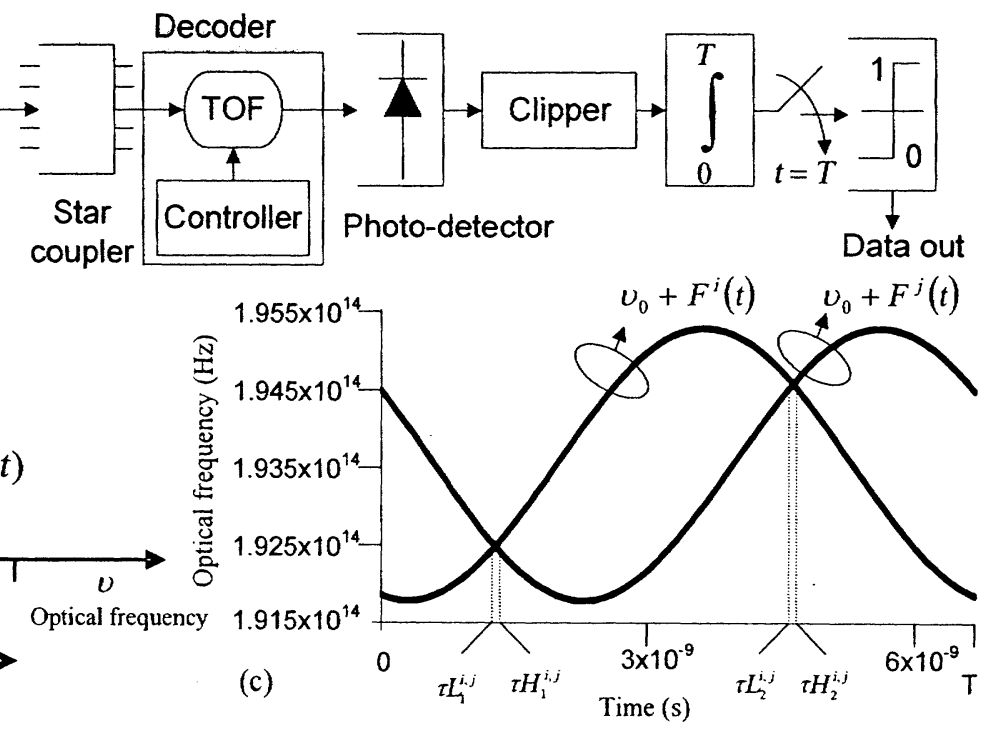

Fig. 1. (a) Block diagram of Dynamic OCDMA system. (b) Optical spectrum of a signal from one of the users. (c) Power spectral density for two users as a function of time and frequency.

TOFs of the decoders are synchronized in time with a phase shift related to the functional code for each one of them. The output of the decoder is therefore the signal which has the same phase shift with some interference noise at the points of intersection with other users.

\section{CODE CONSTRUCTION}

The main criterion in the functional codes construction is to minimize the number of intersecting points between any pair of functions since they increase the interfering power between users. The area of intersection between any two functions which is related directly to the value of interfering power is also an important parameter in the construction of the functional codes. In our proposal, we suggest the use of shifted sine functions to alter the optical central frequency $\left(v_{0}\right)$ for coding which is given by,

$$
F^{j}(t)=(\Delta v / 2) \sin (2 \pi f t-j \varphi)
$$

where $f$ is the frequency of the functional code, and $\varphi$ is the phase shift between different functions. Sine shifted functions are proposed for their simplicity to prove the concept and the ease of achieving the large number of codes required by reducing the phase shift.

The speed of the TOF and its controller required is directly proportional to the amplitude and frequency of the functional code. Thus, it should also be considered in the code construction. Furthermore, the functional codes should start and stop at the same central wavelength during the bit period $\mathrm{T}$ for smooth modulation of the TOF and its controller. For these reasons we choose the smallest frequency that achieves the requirements for the code family which equals to the data bit rate. Phase shift is chosen taking into consideration the spacing between users and the code cardinality. Smaller phase shift results in larger family of codes, but it reduce the spacing between users in the spectrum. The phase shift is chosen to be $2 \pi / 170$, that gives a 170 maximum number of different codes which is close to the cardinality of MQC code with $p=13$ proposed in [2].

\section{DYNAMIC OCDMA PERFORMANCE ANALYSIS}

The variance of photocurrent detected from unpolarizéd thermal light source generated by spontaneous emission including the effect of MAI can be expressed as,

$\sigma_{t}^{2}=(K-1) \sigma_{D A I}^{2}+I^{2} B \tau_{c}+4 K_{b} T_{b} B / R_{L}$

where $(K-1) \sigma_{D A}^{2}$ is the variance of the MAI, $\sigma_{D A}^{2}$ is the variance of the interference when two users access the network, I is the average photocurrent, $B$ is the noiseequivalent electrical bandwidth of the receiver, $\tau_{c}$ is the coherence time, $K_{b}$ is the Boltzmann's constant, $T_{n}$ is the absolute receiver noise temperature, and $R_{L}$ is the receiver load resistor. The first term of this equation represent the MAI effect, the second term denotes the effect of PIIN, and the third term represents the effect of thermal noise.

The power spectral density $G(v, t)$ of the signal at the input of receiver $m, m \in\{1,2, \ldots K\}$ is the sum of all active users' transmitted signals,

$G_{m}(v, t)=\frac{P_{r}}{\Delta v} \sum_{j=1}^{K} b^{j} \operatorname{rect}\left(\frac{v-v_{0}-F^{j}(t)}{B W}\right)$

where $\operatorname{rec}\left(\frac{v-v_{0}}{B W}\right)=v\left(v-v_{0}+\frac{B W}{2}\right)-v\left(v-v_{0}-\frac{B W}{2}\right), u(v)$ is the unit step function, $B W$ is the Bandwidth of the TOF's, and $b^{j}$ is the data bit value of user $j$.

The receiver applies a synchronized matched filter in decoding the incoming signal to extract the desired users data bit stream. The decoder output is, 


$$
\begin{aligned}
G_{m}(v, t)= & \frac{P_{r}}{\Delta v} b^{m} \operatorname{rect}\left(\frac{v-v_{0}-F^{m}(t)}{B W}\right)+\left(\frac{P_{r}}{\Delta v} \sum_{j=1, j \neq m}^{K} b^{j}\right. \\
& \left.\operatorname{rect}\left(\frac{v-v_{0}-F^{j}(t)}{B W}\right)\right) \operatorname{rect}\left(\frac{v-v_{0}-F^{m}(t)}{B W}\right)
\end{aligned}
$$

Then, the photocurrent is,

$$
\begin{aligned}
I_{\mathrm{m}}(t)= & \Re \int_{v=0}^{\infty} G_{m}(v, t) d v \\
= & \Re \frac{P_{r}}{\Delta v} b^{m} B W+\Re \frac{P_{r}}{\Delta v} \sum_{j=1, j \neq m}^{K} b^{j} \\
& \sum_{i=1}^{N_{-j}}\left(B W-\left|F^{m}(t)-F^{j}(t)\right|\right)\left(u\left(t-\tau L_{i}^{m, j}\right)-u\left(t-\tau H_{i}^{m, j}\right)\right)
\end{aligned}
$$

where $\mathfrak{R}=(\eta \mathrm{e}) /\left(h v_{0}\right)$ is the responsivity of the photo-detector, here $\eta$ is quantum efficiency, $e$ is the electron's charge, $h$ is Planck's constant, $N_{m, j}$ is the number of intersecting points between users $m$, and $j$ during one bit period, and $\tau L_{i}^{m, j}, \tau H_{i}^{m, j}$ defined as the roots of the following equations respectively (see Fig. 1(c)),

$$
\begin{aligned}
& F^{m}(t)-F^{j}(t)-B W=0 \\
& F^{m}(t)-F^{j}(t)+B W=0
\end{aligned}
$$
is:

After the integrator and sampler, the optical photocurrent

$$
\begin{aligned}
& I_{m}=\frac{1}{T} \int_{t=0}^{T} I_{m}(t) d t \\
& =\mathfrak{R} b^{m} \frac{P_{r}}{\Delta v} B W+\mathfrak{R} \frac{P_{r}}{T \Delta v} \sum_{j=1, j \neq m}^{K} b^{j} \\
& \sum_{i=1}^{N_{*-j}}\left(B W\left(\tau H_{i}^{m, j}-\tau L_{i}^{m, j}\right)-\int_{I_{j}^{m}, j}^{\tau H^{m . j}} F^{j}(t)-F^{m}(t) \mid d t\right)
\end{aligned}
$$

The optical photo-current at the receiver of user $m$ $m \in\{1,2, \ldots K\}$ after the integrator and sampler can be reformulated as,

$$
I_{m}=b^{m} I+M A I(m)
$$

where $I=\Re B W P_{r} / \Delta v$, and the multiple access interference at receiver $m, M A I(m)$ is given by,

$$
\operatorname{MAI}(m)=\sum_{j=0, j \neq m}^{K} b_{j} D A I(m, j),
$$

where,

$$
\begin{aligned}
\operatorname{DAI}(m, j)= & \mathfrak{R} \frac{P_{r}}{T \Delta v} \\
& \sum_{i=1}^{N_{m, j}}\left(B W\left(\tau H_{i}^{m, j}-\tau L^{m, j}{ }_{i}\right)-\int_{\tau L^{\infty}, j}^{\tau H_{i}^{-j, j}} F^{j}(t)-F^{m}(t) \mid d t\right)
\end{aligned}
$$

is the interference between users $m$ and $j$.
In equation (8), the first term is the data bit of the desired user $m$, and the second term is the MAI Noise.

To reduce the MAI and improve the performance of the DOCDMA system, an electrical clipper is placed at the output of the photo-detector to clip the MAI noise in time domain. An electrical clipper is defined as,

$$
g(t)=\left\{\begin{array}{cc}
x, & t \geq x \\
0 & 0 \leq t<x
\end{array}\right.
$$

where $x$ is threshold value of the clipper. As a result, any electrical signal of more than $x$ will be clipped back to $x$, while any signal with a photo-current of less than $x$ will have a response of zero at the clipper output.

At the output of the clipper with a threshold value of $x$, the signal can be given as,

$$
\begin{aligned}
I_{m o}^{x<I}(t)= & g\left(I_{m}(t)\right)=b^{m} x+\overline{b^{m}} x \\
& \sum_{j=1, j \neq m}^{K} b^{j} \sum_{j=1}^{N_{m, j}}\left[u\left(t-\tau 1_{i}^{m, j}\right)-u\left(t-\tau 2_{i}^{m, j}\right)\right]
\end{aligned}
$$

where $I_{m}(t)$ is the photo-current at the output of the decoder. $\tau 1_{i}^{m, j}$ and $\tau 2_{i}^{m, j}$ are defined as the roots within the time period from $\tau L_{i}^{\mathrm{m}, j}$ to $\tau H_{j}^{\mathrm{m}, j}$ of equation (13) and (14) respectively,

$$
\begin{aligned}
& R 1(t)=\Re \frac{P_{r}}{\Delta v} b^{j}\left(B W-F^{m}(t)+F^{j}(t)\right)-x \\
& R 2(t)=\Re \frac{P_{r}}{\Delta v} b^{j}\left(B W-F^{j}(t)+F^{m}(t)\right)-x
\end{aligned}
$$

The noise when the transmitted signal is zero is the important part that affects the bit error rate performance. The clipped photo-current passes though an integrator and sampler could be defined as,

$I_{\mathrm{mc}}=\frac{1}{T} \int_{t=0}^{T} I_{\mathrm{mo}}^{\kappa I}(t) d t=b^{\mathrm{m}} X+\frac{\overline{b^{m}} X}{T} \sum_{j=1, j \neq m}^{K} b^{j} \sum_{j=1}^{N_{m, j}}\left(\tau 2_{i}^{m, j}-\tau 1_{i}^{m, j}\right)$

This Equation could be reformulated as,

$I_{m c}=b^{m} X+M A I_{c}(m)$

The MAI after clipping is given by,

$M A I_{c}(m)=\sum_{j=0, j \neq m}^{K} b_{j} D A I_{c}(m, j)$

where,

$D A I_{c}(m, j)=\overline{b_{m}} \frac{x}{T} \sum_{i=1}^{N_{m, j}}\left(\tau 2_{i}^{m, j}-\tau 1_{i}^{m, j}\right)$

Since our system is synchronized, users $m$ and $j$ will interfere at the same points in time relative to the beginning of the bit period, and the intersecting edges $\tau L^{m, j}{ }_{i}$ and $\tau H^{m, j}$ are the same whenever users $m$ and $j$ are active. This results 
in a constant value of $\operatorname{DAI}(m, j)$ if users $m$ and $j$ are active, otherwise $\operatorname{DAI}(m, j)$ is zero. With signal clipping, only MAI can pass through if user $m$ is sending a data bit with value of " 0 and user $j$ is sending data bit with value of " 1 . For equi-probable data, $\operatorname{DAI}(m, j)$ is random variable with variance, with average and variance given in (19) and (20) respectively,

$$
\begin{aligned}
\mu_{D A I} & =\frac{1}{K^{2}-K} \sum_{m=1}^{K} \sum_{j=1, j \neq m}^{K} D A I(m, j) \\
\sigma_{D A I}^{2} & =\frac{1}{K^{2}-K} \sum_{m=1}^{K} \sum_{j=1, j \neq m}^{K}\left(D A I(m, j)-\mu_{D A l}\right)^{2}
\end{aligned}
$$

The variance of MAI can be approximated as $(k-1) \sigma_{D A I}^{2}$ for $k$ simultaneous active users.

The PIIN causes variations in the output current during interference of incoherent light sources at the input of photo-detector. The variance of the PIIN is related to the coherence time of the source $\left(\tau_{c}\right)$, as given in Equation (2), which is given by,

$$
\tau_{c}(t)=\left(\int_{v=0}^{\infty} G_{m}^{2}(v, t) d v\right) /\left(\int_{v=0}^{\infty} G_{m}(v, t) d v\right)^{2}
$$

Assuming no more than one pair of users interfering at the same time, which is the case as in our proposed functional code family and averaging the variance at the points of interference along the bit period and averaging over all users, the variance of PIIN is given by,

$$
\begin{aligned}
\sigma_{P \mathbb{N}}^{2}= & \frac{1}{K} \sum_{m=1}^{K} \frac{1}{T} \int_{0}^{T} B R^{2} \sum_{j=1, j \neq m}^{K} \sum_{j=1}^{N_{x i}}\left(\left(\frac{P_{r}}{\Delta v} b_{m}+\frac{P_{r}}{\Delta v} b_{j}\right)^{2}\right. \\
& \left.\left(B W-\left|F^{m}(t)-F^{j}(t)\right|\right)+\left(\frac{P_{r}}{\Delta v} b\right)^{2}\left|F^{m}(t)-F^{j}(t)\right|\right) \\
& \left(u\left(t-\tau L_{i}^{m j}\right)-u\left(t-\tau H_{i}^{\mathrm{m} j}\right)\right) d t
\end{aligned}
$$

\section{RESULTS AND DISCUSSION}

The total variance is calculated for the clipped DOCDMA system with shifted sine functional code family proposed. Fig. 2 shows the relation between the BER and the number of simultaneous active users when $P_{r}=-10 \mathrm{dBm}$. The parameters used in the calculation are: $\Delta v=30 \mathrm{~nm}$, $v_{0}=1550 \mathrm{~nm}, B R=155 \mathrm{Mbps}$, and filter bandwidth of $B W=0.165 \mathrm{~nm}$ which is equal to the chip width of SAC system using MQC with prime number of 13 and the same optical bandwidth. The BER functions for DOCDMA system without signal clipping and another two SAC systems, one using Hadamard code and the other using MQC code with prime number of 13 [5], are also plotted in the same figure for the sake of comparison. The proposed system has a significant improvement over that without clipping. For an error rate of $10^{-13}$, it can accommodate up to 160 users, whereas for other systems, the maximum simultaneous users are 60 for DOCDMA without clipping,
50 for SAC system using MQC code, and 30 for SAC system using Hadamard code. The BER of the DOCDMA system is increasing at a slower rate than that of the other two systems, which indicates that there is a significant improvement in performance at large number of users. Indeed it is shown that the BER for DOCDMA is better at any number of users of more than 45 . However, for less than 45 active users, SAC system with MQC gives BER better performance than that of DOCDMA system without clipping. It should be noted that for this range of users, the error rate is too small (less than $10^{-15}$ ). Signal clipping enhances the performance of the DOCDMA system by reducing the BER for any number of users.

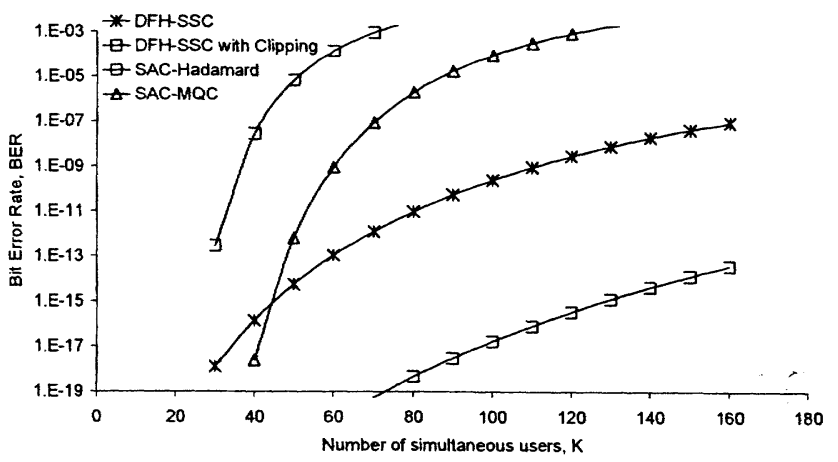

Fig. 2: Probability of error versus simultaneous active users when $P_{r}=-10 \mathrm{dBm}$

\section{CONCLUSION}

We have proposed a novel low noise clipped optical dynamic CDMA communication system. The encoder/decoder design is based on fast tunable optical filter. The filters are controlled dynamically during the data bit period. Dynamic OCDMA with sine shifted functional (other functions can also be used) code family is analyzed taking into account MAI, PIIN, and thermal noise. The system improves the BER performance when the signal clipping is used, and small BER at large number of simultaneous active users compared to other systems like SAC-CDMA systems using Hadamard and MQC codes. The interference effect on performance of the system is reduced by clipping the signal at the output of the detector.

\section{REFERENCES}

[1] J. A. Salehi, C. A. Brackett, "Code division multiple-access techniques in optical fiber networks. II. Systems performance analysis," IEEE Trans. on Communications, Vol. 37, Issue 8, pp. 834842, Aug. 1989.

[2] Zou Wei, H. M. H. Shalaby, H. Ghafouri-Shiraz, "Modified quadratic congruence codes for fiber Bragg-grating-based spectral-amplitudecoding optical CDMA systems," Journal of Lightwave Technology, Vol. 19, Issue 9, pp. 1274-1281, Sept. 2001.

[3] Zou Wei, H. Ghafouri-Shiraz, H. M. H. Shalaby, "New code families for fiber-Bragg-grating-based spectral-amplitude-coding optical CDMA systems," IEEE Photonics Technology Letters, Vol. 13, Issue 8, pp. 890-892, Aug. 2001.

[4] D. Zaccarin, M. Kevehrad, "An optical CDMA system based on spectral encoding of LED," IEEE Photonics Technology Letters, Vol. 4, pp. 479-482, Apr. 1993.

[5] D.Sadot, E. Boimovich, "Tunable optical filters for dense WDM networks," IEEE Communications Magazine, Volume. 36, Issue 12, pp. 50-55, Dec. 1998 